\title{
Retrospective analysis of HSG in primary infertility
}

\author{
Mishra R. K. ${ }^{1}$, Dave P. K. ${ }^{2}$, Jain M. ${ }^{3}$, Malini B. ${ }^{4}$, Jain S. ${ }^{5}$ \\ ${ }^{1}$ Dr. R. K. Mishra, Associate Professor, ${ }^{2}$ Dr. P. K. Dave, Assistant Professor, ${ }^{3}$ Dr. M. Jain, Department of \\ Radiodiagnosis, ${ }^{4}$ Dr. Malini Bhardwaj, ${ }^{5}$ Dr S. Jain, Department of Obstetrics and Gynecology, all authors are \\ affiliated with L. N. Medical College and J. K. Hospital, Kolar Road, Bhopal (M P.) India.
}

Address for Correspondence: Dr. Pranav K. Dave, Assistant Professor, Department of Radiodiagnosis, L.N. Medical College and J. K. Hospital, Kolar Road, Bhopal, MP. India. E-mail: pranavkdave@rediffmail.com

\begin{abstract}
Introduction: Hysterosalpingography used to be a common Radiological Investigation since long time for the investigation of infertility. In the current era of imaging HSG remains to be a choice of investigation for infertility cases. It provides anatomical depiction of uterus and fallopian tubes by introduction of contrast agent. Objective: Objective of this study is to analyze the common pathological conditions detected on HSG in cases of primary infertility. Methodology: 140 cases of HSG were reviewed retrospectively in cases of primary infertility. Study covered age ranging from $20 \mathrm{yrs}$ to $42 \mathrm{yrs}$. The largest number of patients who reported were between the age of $26-30$ years $-47.14 \%$. Maximum patients were in the marital duration of 2-5 yrs $-65 \%$, followed by 6-10 Yrs $-24 \%$ and remaining $16 \%$ above the duration of more than 10 yrs. Routine use of contraceptives by both partners were excluded in this study. Infertility caused by the male factor was also excluded. Standard protocol for HSG was followed with use of water soluble contrast. Results: Analysis of 140 cases show common cause of infertility as congenital uterine anomalies $-6.42 \%$, various levels of tubal blocks17.2\%, Uterine SOL/ Fibroid- 4.28\% and pelvic adhesions- 6.42\%. Conclusion: Hysterosalpingography still remains the choice of investigation in cases of primary infertility. Most of the cases with primary infertility had a normal HSG, followed by various uterine congenital anomalies and fallopian tube obstruction with or with hydrosalpinx. HSG is repeatable, cost effective, safe and less time-consuming procedure except for the fact that it involves small X-ray exposure to patient.
\end{abstract}

Key words: Hysterosalpingography, Infertility, uterus

\section{Introduction}

Hysterosalpingography delineates the female reproductive organs. It is in practice since over 50 years in clinical gynaecology for evaluation of cause of primary infertility. According to WHO the primary infertility is defined as if the couple has never conceived despite cohabitation and exposure to pregnancy, without use of any contraceptive device for a period of two years. WHO states that $50-80$ million of couple are infertile globally. In India the number was $8 \%$ of the population in 1981 and showing rising trend since then [1,2]. HSG evaluates female genital tract and radiographically delineates uterine and tubal cavity [3]. In radiological practice number of $\mathrm{HSG}$ procedure

Manuscript received: $18^{\text {th }}$ August 2017

Reviewed: $28^{\text {th }}$ August 2017

Author Corrected: $4^{\text {th }}$ September 2017

Accepted for Publication: $9^{\text {th }}$ September 2017 have increased in last few years due to advances in reproductive medicine and tendency of females for delaying childbearing [4]. HSG provides good morphology of fallopian tubes and uterine cavity with emphasis on tubal patency [5,6]. Properly performed HSG gives accurate information about endo-cervical canal, diameter, configuration of internal canal, location and direction of fallopian tubes, status of fimbriae and spillage of contrast into peritoneal cavity. Interpretation requires cautions as under or over read may lead to mismanagement [7,8]. By making the correct diagnosis patients of infertility are benefited [4,9].

\section{Methodology}

Study design and setting- Study was conducted in tertiary care teaching hospital of central India. It 
included retrospective analysis of HSG carried out in cases of primary infertility referred to the department from December 2015 to July 2017.

A detail personal history was obtained regarding duration of married life, cohabitation, use of contraceptives and exposure to pregnancy.

Inclusion criteria- Only cases having primary infertility as per WHO criteria reported for HSG to the department.

\section{Exclusion criteria}

- Cases having secondary infertility.

- Cases using contraceptive methods during this period.

- Infertility caused by male factor.
Original Research Article

After due written consent from the patient for the procedure, HSG was carried out as per standard protocol that includes timing as per ten-day rule after the first week of menses. After required premedication and taking aseptic precautions, water soluble contrast was introduced trough the canula under fluoroscopic guidance. Skiagrams were obtained during the phase of uterine cavity filling and subsequently to watch for the tubal patency and spillage in peritoneal cavity. Delayed skiagram was obtained for confirmation of hydrosalpinx. Independent interpretation of the procedure was obtained by two radiologists. No untoward reaction was found in our series.

Data collection and analysis- Radiological data were entered. SPSS 11.0 software programs were used for analysis.

\section{Results}

In the study period 140 cases of HSG were included in study.

Marital duration: Maximum cases of the marital duration were between 2-10 Years $-82 \%$, with the peak of 25 Years $-62 \%$.

Table-1: Age distribution of patients in our study.

\begin{tabular}{|c|c|c|}
\hline Age in Years & Number of cases & Percentage \% \\
\hline $20-25$ & 35 & $25 \%$ \\
\hline $26-30$ & 66 & $47.14 \%$ \\
\hline $31-35$ & 27 & $19.28 \%$ \\
\hline 36 and above & 12 & $8.57 \%$ \\
\hline
\end{tabular}

The analysis of above study shows that the commonest age group reported was between the ages of $20-30$ yrs$71.14 \%$ with peak range at $26-30 \mathrm{yrs}-47.14 \%$. Rest were above the age of $31-35 \mathrm{yrs}-19.28 \%$ and above 36 yrs $-8.57 \%$.

Table-2- Various uterine pathology patterns observed.

\begin{tabular}{|c|c|c|}
\hline Findings & Number of cases & Percentage \% \\
\hline Normal & 123 & 87.8 \\
\hline Uterine congenital anomalies & 9 & 6.42 \\
\hline Uterine Inflammatory lesions & 3 & 2.14 \\
\hline SOL (fibroid) & 6 & 4.28 \\
\hline Syneacae & 1 & 0.71 \\
\hline Pelvic adhesion & 9 & 6.42 \\
\hline Venous intravasation & 3 & 2.14 \\
\hline
\end{tabular}

In $87.8 \%$ cases the uterine cavity was normal while $6.42 \%$ showed various congenital anomalies. Uterine inflammatory lesions were observed in $2.14 \%$ cases, Space occupying lesions (Fibroids) were seen in $4.28 \%$, syneachae in $0.71 \%$ and pelvic adhesion in $6.42 \%$ cases. Venous intravasation of contrast was noted in $2.14 \%$ cases in our series. 
Table-3: Various tubal pathology patterns observed.

\begin{tabular}{|c|c|c|}
\hline Findings & Number of cases & Percentage \% \\
\hline Right proximal block & 4 & 2.85 \\
\hline Right distal block & 1 & 0.71 \\
\hline Left proximal block & 3 & 2.14 \\
\hline Left Distal block & 1 & 0.71 \\
\hline Bilateral block & 9 & 6.42 \\
Proximal & 7 & 5 \\
Distal & 2 & 1.42 \\
\hline Hydrosalpinx with block on Right side & 2 & 1.42 \\
Left side & 3 & 2.14 \\
Both sides & 3 & 2.14 \\
\hline Only right sided block with P/H - salpingectomy & 1 & 0.71 \\
\hline Bilateral Normal & 116 & 82.8 \\
\hline Only left normal & 5 & 3.57 \\
\hline Only right normal & 4 & 2.85 \\
\hline
\end{tabular}

Unilateral Right proximal level blocks were observed in $2.8 \%$, Right distal level blocks in $0.7 \%$. Unilateral left proximal level blocks in 2.14\% and Left distal level block in $0.7 \%$. Bilateral tubal blocks were seen in $6.42 \%$. (Proximal 5\% \& distal 1.42\%). Hydrosalpinx with block on Right side was seen in 1.4\% while on Left side $2.14 \%$. Hydrosalpinx with bilateral block was observed in $2.14 \%$. In one case right sided block was seen due to Past History of salpingectomy (0.7\%). Tubes were Normal bilaterally in $82.8 \%$ cases.

\section{Discussion}

Infertility is one of the most common problem in women`s health in developing countries. Poverty, pollution, lack of education and awareness, gender inequality and infectious diseases are major contributing factors.

HSG was performed following the menses considering 10 days rule as the isthmas is at its most distensible phase and fallopian tubes are most readily filled by the contrast media during this period. HSG was not performed in the second half of the cycle because of the fear of inadvertently radiating the ongoing pregnancy if any and because of the thickened endometrium which could lead to false positive block of proximal part of the tubes $[8,9,10]$.

The analysis shows that the commonest age group affected was between the ages of $20-30 \mathrm{yrs}-71.14 \%$ with peak range at $26-30 \mathrm{yrs}-47.14 \%$. This age group coincides with the most active reproductive period in female. Maximum cases of the marital duration were between 2-10 Yrs - 82\%, with the peak of 2-5 Yrs -62\%. These findings correlate well with the previous study [3,7]. HSG is an established procedure for evaluation of morphology of uterus and fallopian tubes $[11,12,13]$.

Analysis of our study shows bilateral tubal block in 9 patients, followed by right tubal block in 5 patients and left tubal block in 4 patients. In our study there were equal distribution of unilateral and bilateral tubal block. This findings are in contrast with the earlier finding of Joseph Bako Igashi et al [3]. Majority of our Hydrosalpinx was observed on both sides in 3 cases, only on left side in 3 cases and only on right side in 2 cases. There was a case of right tubal block with past history of salpingectomy while left tube was patent. Tubal block is most likely due to infection which may be in subclinical stage in most of the cases and especially sexually transmitted disease. Chronically inflamed appendix may lead to tubal block. The appendicular inflammation may remain in subclinical stage because of the current use of antibiotics $[3,4,9]$. In early stage of infection tubal patency is not affected but delaying in treatment lead to total block. 


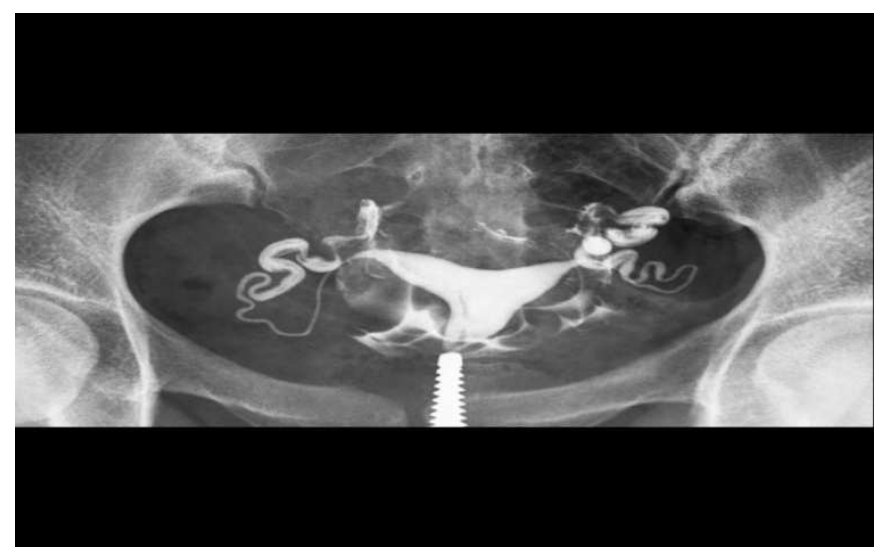

Fig-1: Normal HSG late filling and spillage phase

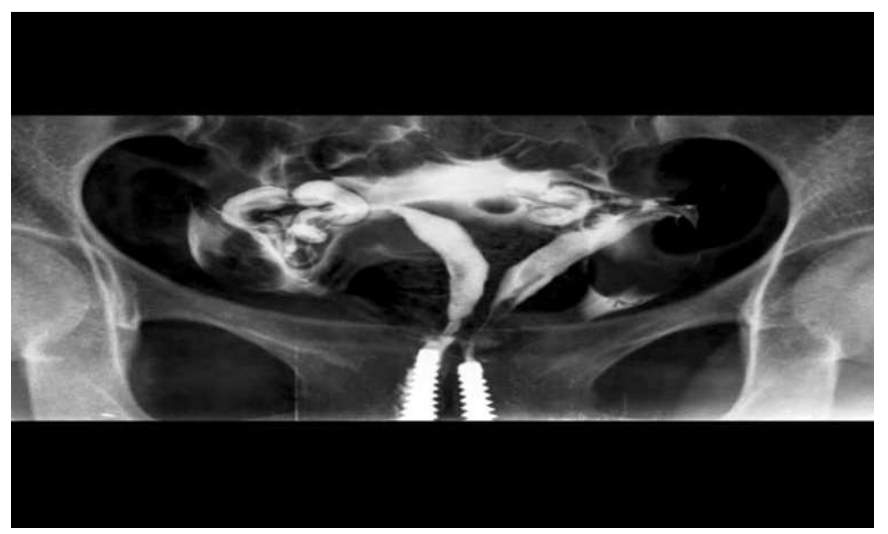

Fig-2: Uterus bicornisbicollis case 1

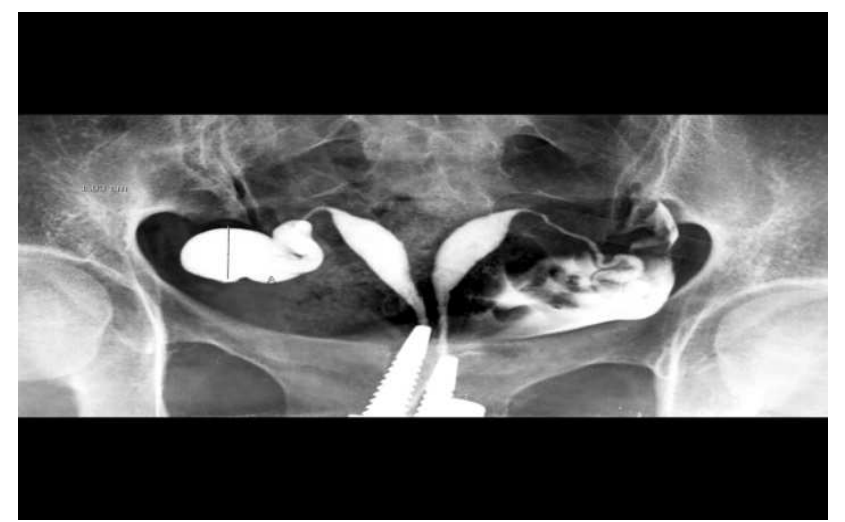

Fig-3: Uterus bicornisbicollis with hydrosalpinx right

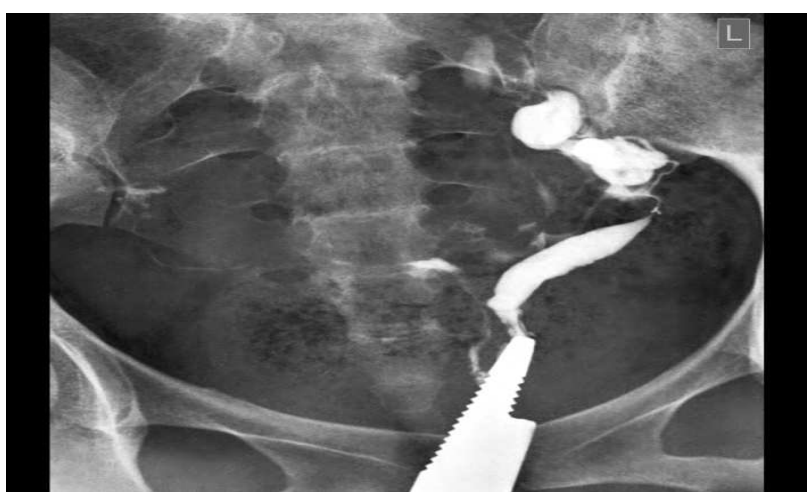

Fig-4: Unicornuate uterus with normal spillage left 
Uterine SOL/fibroid was the next common uterine abnormality noted in our study accounting for 6 cases (4.28\%). Incidence of uterine fibroids in the study of Joseph Bako Igashi et al [3] was 16\% which shows that the incidence of uterine fibroids were less in our study. Leiomyomas or fibroids are benign tumours arising from smooth muscles of the uterus [14]. The exact etiology is unknown however it is diagnosed in younger age group and more common in black races. [4, 14]. Fibroids are estrogen dependent and decrease in size with age. Fibroids are of different types depending on their location such as intramural, submucosal and subserousal. Rarely they may be pedunculated.

Pelvic adhesions were noted amongst the peritoneal abnormalities which are depicted as delayed or localized spill, lack of tubal mobility and abnormality in tubal contour. Laparoscopy is better for extrinsic tubal pathology evaluation as compared to HSG. [15,16]. Hysterosalpingography is not the investigation of choice for visualization of ovaries however ovaries are enlarged could be seen when a large quantity of contrast material freely spilled surrounding the ovaries in peritoneal cavity. [17]. Syneachae was seen in only one case in our study group. This could be result of improperly treated infection. [17]. Venous intravasation of the contrast were seen in 3 cases. Venous intravasation could occur due to accidental injection of contrast into endometrium which could be due excess injection pressure, trauma to the endometrium by the tip of canula and if the examination is performed during the deficient endometrium thickness. It is harmless but at times it obscures the optimal evaluation of HSG. In our series 9 cases $(6.42 \%)$ conceived immediately following the HSG procedure.

This could mean that fallopian tube patency was improved after the HSG procedure [12]. We have not encountered any case of peritoneal irritation caused by the use of water soluble contrast material spilling into the peritoneal cavity. This finding concurs with the study of Heun Y. Yune [17]. Population control has also played an adverse roll. Children in the family are associated with happiness, wellbeing and social security, particularly in Indian society. Childless couple often leads to adverse social and societal consequences [18].

\section{Conclusion}

Hysterosalpingography still remains the choice of investigation in cases of primary infertility. Most of the cases with primary infertility had a normal HSG. Population studied showed variable causes for primary infertility like various congenital anomalies and fallopian tube obstruction consisting of unilateral or bilateral block with or with hydrosalpinx. Uterine SOL/ fibroid were next finding noticed on HSG. HSG is repeatable, OPD based, cost effective and less time consuming procedure however there is exposure to radiation which combat the benefit to the patient. No untoward reaction was found in our series. This points towards the need for more emphasis on early evaluation and treatment.

\section{Funding: Nil, Conflict of interest: None Permission of IRB: Yes}

\section{References}

1. Paul C. Adamson, Karl Krupp, Alexandra H. Freeman, Jeffrey D. Klausner, Arthur L. Reingold, Purnima Madhivanan. Prevalence and correlates of primary infertility among young women in Mysore, India. Indian J Med Res. 2011 Oct; 134(4): 440446.
2. Niharika Tripathi, Infertility among Indian Women: Emerging Evidence and Need for Policy Measures. International Institute for Population Sciences (I.I.P.S), Mumbai, India. Princeton. edu/ papers/ 110609.

3. Joseph Bako Igashi, Dlama Zira Joseph, Gloria Joseph, Saidu Kadas, Geofrey Luntsi, Flavious Nkubli, Samuel Laushugno, Obetta Chinedu T. Hysterosalpingography: A Re-Emerging Study in Health Care Transformation. IOSR Journal of Dental and Medical Sciences (IOSR-JMDS) Volume 13, Issue 12 Ver. VII (Dec. 2014): $37-40$

4. Mgbor S.O. Patterns of Hysterosalpingography (HSG) Findings in Gyneacology patients in Enugu, Nigerian Medical Journal 2006; 47:14-16

5. Ezegwui H.U., Mgbor S.O, Ikeme A.C, Patients Journal of Obstetrics and Gyneacology.2001. 18:14-16.

6. Ghazi A, Saddique M.S., Saddique N, Jabbar S. Tehmina Ali, Sunita Jaipal, Pakistan Journal of Surgery. 2007 Oct - Dec 23 (4): 283-286. 
7. Asaleye C.M., Adetiloye V.A., Oyinlola T.O. Review of HSG reports done for infertility, Nigerian Journal of Health Sciences: 2004, 4: 36-39.

8. Okogbenin S.A., Okepere E.E., Eigbefoh J.I., Ogbo F.O., Okonta P.I., Onuh S.O. Gynaecological indication for abdominal hysterectomy in the university of Benin teaching Hospital. Archieves of Clinical Journal. 2006.1:56-63.

9. Kawuwa M.B., Maigari A.G., Audu B.M., Indications and implications of hysterectomy in Maiduguri; Kanem Journal of Medical Sciences 2007: 1 (1):20-25.

10. Crofton M. Gyneacological Imaging. Text book of Radiology and Imaging $7^{\text {th }}$ edition. 2007 Vol-2.

11. Good clinical treatment in assisted reproduction - An ESHRE position paper. Eur. Soc. Hum. Reprod. 2008- 255.

12. Johnson N, Vandekerckhove $\mathrm{P}$, Watson A, Lilford R, Harada T, Hughes E. Tubal flushing for subfertility. Cochrane Database Syst Rev. 2005 Apr 18; (2): CD003718.
13. Ezegwui H.U., Mgbor S.O., Ikeme A.C. Patients Journal of Obstetric and Gyneacology. 2001:18:14-16.

14. Incidence and age distribution of uterine fibroid at FMC owerri. Nigeria. Journal of Medical Imaging and Radiation therapy. 2012 Nov. Vol. 1 No.3: 25-29.

15. Akano A.O., Olatunji O.O., Agaja E.O., and Olakoju P.E., Severe Reaction after Buscopan premedication for HSG. Archieves of Nigerian Medicine and Medical Sciences 2009. 1(1): 23-25.

16. Arsian S.A., Itabeys, Koc C, Var T, Bilge U., Evaluation of infertile Women: HSG, Office hysteroscopy or Hysterography: which is better? Journal of infertility 2004; 4:1-4.

17. Heun Y. Yuneetal., Hystereosalpingography in infertility Paper Presented at the $74^{\text {th }}$ annual meeting of American Roentgen Ray Society, Montreal, P.Q., Canada, September 1973; 25-28.

18. Muhammad Usman Aziz, Saleha Anwar. Hysterosalpingographic evaluation of primary and secondary infertility. Pak J Med Sic 2015 SeptOct; 31 (5): 1188-1191.

\section{How to cite this article?}

Mishra R. K, Dave P. K, Jain M, Malini B, Jain S. Retrospective analysis of HSG in primary infertility. Int J Med Res Rev 2017;5(10):894-899.doi:10.17511/ijmrr. 2017.i10.04 